through a material, these components separate - a process called dispersion. There are two types of dispersion: normal and anomalous. In normal dispersion, the low frequencies move faster than the high frequencies ('red leads blue'), whereas in anomalous dispersion, the high frequencies lead the low frequencies ('blue leads red').

However, a high-power beam can cause the change in the material's refractive index to shift the lower frequencies ('red') to the front of the pulse and the higher frequencies ('blue') to the rear. By tuning the beam intensity, the effect of anomalous dispersion can be cancelled out. Furthermore, if a bright beam is turned off and then back on (a dark pulse), the frequency shifts are reversed and normal dispersion can be neutralized.

A $3 \mathrm{D}$ soliton, sometimes referred to as a light bullet, is the result of cancelling out diffraction and dispersion simultaneously (Fig. 1). Although such objects exist in theory, they are notoriously unstable. The focusing of the beam by the material must perfectly balance diffraction, and it is extremely difficult to counteract diffraction and dispersion at the same time, because these actions require different beam intensities. Scientists have generated solitons that are stable in two dimensions (one along the direction of propagation and one perpendicular to this direction $)^{3}$, and $3 \mathrm{D}$ solitons in a highly structured material (glass patterned with an array of optical devices called waveguides) $)^{4}$. But it has not been possible to create $3 \mathrm{D}$ solitons in an unstructured material - which is desirable for studying these objects and for practical applications.

It has been known for more than 20 years that self-guided light beams can be generated in photorefractive materials ${ }^{5}$. These are materials that exhibit a temporary change in refractive index when exposed to a beam of light, as a result of electrons moving through them. The focusing of the beam occurs in such a way that the need for perfect control of the beam intensity is eliminated. Furthermore, bound electrons produce the frequency-shifting refractive index required to eliminate dispersion. There is only one hitch: the material needs to have regions of negative and positive electric charge, but such a charge distribution takes time to establish - longer than the duration of a short light pulse.

Lahav and colleagues' solution was to shine a repetitive string of such pulses into a crystal of the photorefractive material strontium barium niobate, which responded to the power averaged over many pulses to create a self-guided beam. The response of the bound electrons in the crystal then allowed dispersion to be cancelled out in each pulse. The result was a string of 3D 'pulse-train' solitons - so named because the properties of each soliton depend on the solitons that come before it.

The authors used pulses of 800-nanometre wavelength, which meant that the crystal had normal dispersion. As a consequence, although the beam produced was bright, its temporal profile consisted of a dark pulse (see Figure 2 of the paper ${ }^{2}$ ). By performing similar experiments at longer wavelengths, for which the crystal has anomalous dispersion, it should be possible to generate bright $3 \mathrm{D}$ solitons - one of the major goals in the field of nonlinear optics.

Considering the difficulty in controlling localized 3D wave packets, Lahav and colleagues' results constitute a substantial advance. Interest in localized but non-spreading 3D wave packets extends well beyond optics, to areas as disparate as exotic states of matter known as Bose-Einstein condensates ${ }^{6}$ and excitations of substances called ferromagnetic colloids ${ }^{7}$. Furthermore, it should now be possible to investigate how 3D solitons interact when they collide - do they pass right through each other, interact or merge? This information might be useful some day for optical information processing ${ }^{8}$.

Frank W. Wise is in the School of Applied and Engineering Physics, Cornell University,

Ithaca, New York 14853, USA.

e-mail:frank.wise@cornell.edu

1. Kivshar, Y. S. \& Agrawal, G. Optical Solitons: From Fibers to Photonic Crystals (Academic, 2003).

2. Lahav, O. et al. Phys. Rev. X 7, 041051 (2017).

3. Liu, X., Qian, J. \& Wise, F. W. Phys. Rev. Lett. 82, 4631-4634 (1999)

4. Minardi, S. et al. Phys. Rev. Lett. 105, 263901 (2010).

5. Shih, M. et al. Opt. Lett. 21, 324-326 (1996).

6. Strecker, K. E., Partridge, G. B., Truscott, A. G. \& Hulet, R. G. Nature 417, 150-153 (2002).

7. Ackerman, P. J. \& Smalyukh, I. I. Nature Mater. $\mathbf{1 6}$ 426-432 (2017).

8. McLeod, R., Wagner, K. \& Blair, S. Phys. Rev. A 52, 3254-3278 (1995).

This article was published online on 5 February 2018.

\title{
Smoking gun for a rare mutation mechanism
}

\section{In 1953, James Watson and Francis Crick proposed that rarely formed isomers of DNA bases cause spontaneous mutations to occur during the copying of DNA. Sixty-five years later, it looks as though they were right. SEE ARTICLE P.195}

\section{MYRON F. GOODMAN}

$\mathrm{H}$ ow do mutations arise when DNA is copied in cells? On page 195, Kimsey et al. ${ }^{1}$ combine observations of DNA structure with measurements of enzyme kinetics and computational modelling to provide a definitive explanation of a seminal mechanism.

The elucidation of the structure of DNA reported in James Watson and Francis Crick's classic 1953 paper $^{2}$ is a monumental piece of work. The key finding was that DNA has a double-helix structure held together by specific interactions between pairs of bases, now known as Watson-Crick pairs: adenine (A) pairs up with thymine $(\mathrm{T})$, whereas guanine $(\mathrm{G})$ pairs with cytosine $(\mathrm{C})$. The DNA bases exist as tautomers (readily interconvertible pairs of isomers), and the A.T and G.C base pairs contain each base in its predominant tautomeric form (Fig. 1a). The process by which DNA is assembled was not known at the time, but the discovery of base-pairing suggested that the sequence of nucleotides on one strand of a double helix could govern the sequence that was constructed on the complementary strand $^{2}$.

The DNA structure had other far-reaching implications: it suggested a model for how mutations might arise spontaneously as DNA is made. Watson and Crick proposed ${ }^{3}$ that mutations could occur because of "a base occurring very occasionally in one of the less likely tautomeric forms, at the moment when the complementary chain is being formed". In other words, G.T and A.C mispairs could occur if one of the bases is in a disfavoured tautomeric form (Fig. 1b). Such mutations would be easily accommodated because tautomeric mispairs do not distort the helical DNA structure. The disfavoured-tautomer model for spontaneous mutation formation (mutagenesis) was rapidly adopted by biologists and included in textbooks, despite the absence of supporting experimental evidence.

Mispaired structures other than those associated with tautomerization were discovered in the mid-1960s; these included the wobble pairs ${ }^{4}$ proposed by Crick, and Hoogsteen pairs ${ }^{5}$. In the mid-1980s, mispairs associated with charged forms of DNA bases were also identified ${ }^{6-8}$. But it wasn't until 2011 that a C.A mismatch associated with a rare tautomer was finally observed in an X-ray crystal structure ${ }^{9}$. The mismatch was formed between bases of nucleotides bound in the active site of DNA polymerase (the enzyme that synthesizes DNA from nucleotides) when DNA synthesis was performed in the presence 
a

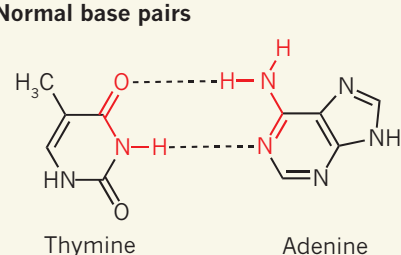

b Mispairs containing rare tautomers
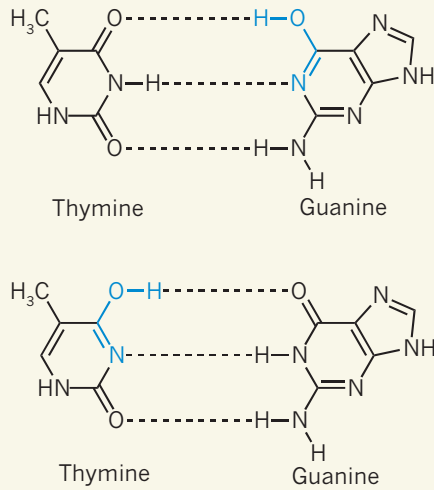
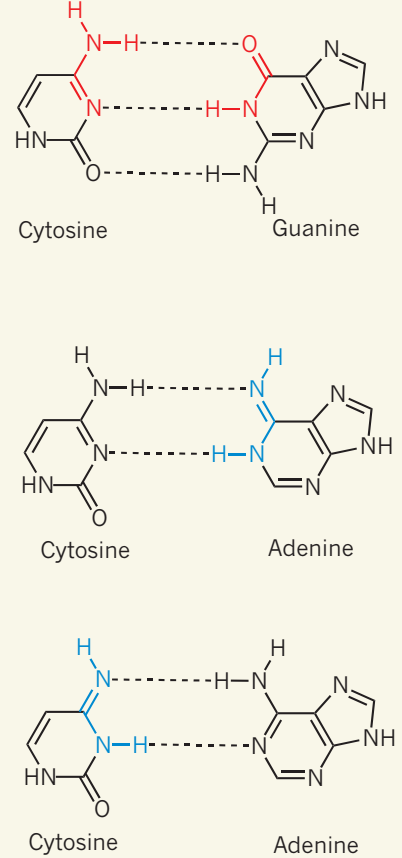

Figure 1 | Base-pair structures in DNA. a, The double-helix structure of DNA is held together by specific interactions (dotted lines) between pairs of bases: adenine (A) pairs with thymine (T), and guanine $(\mathrm{G})$ pairs with cytosine $(\mathrm{C})$. b. The DNA bases form rare isomeric structures known as tautomers, which can allow the formation of mispairs; bonds shown in blue are the tautomeric forms of the bonds shown in red in a. Kimsey et al. ${ }^{1}$ have detected tautomeric G.T mispairs in DNA duplexes, and conclude from modelling studies that this explains the frequency with which G.T is misincorporated into DNA during DNA duplication by polymerase enzymes - as proposed ${ }^{3}$ by Watson and Crick in 1953.

of manganese(II) ions, which are known to cause mutations. A second X-ray structure ${ }^{10}$ reported that year identified an ionized G.T mismatch, also formed between substrates bound by DNA polymerase. In both cases, the mismatched pairs had the same geometry as Watson-Crick pairs.

In Watson and Crick's model for mutagenesis, the rare occurrence of disfavoured tautomeric bases could account for the observed frequency with which DNA polymerases produce mismatches (about one per thousand to one per million base pairs formed $\left.{ }^{11}\right)$. But such tautomers and the associated base pairs were thought to be almost impossible to detect in duplexes. Then, in 2015, 62 years after the mutagenesis model was proposed, researchers from the same group as Kimsey et al. reported a tour de force of experimental work: they used nuclear magnetic resonance (NMR) spectroscopy to identify ${ }^{12}$ a long-lived wobble G.T structure that was in a dynamic equilibrium with transient, rarely formed G.T mispairs associated with disfavoured tautomers, and with ionized G. $\mathrm{T}^{-}$structures, both of which have Watson-Crick geometry.

The first step of the DNA-synthesis process that forms a G.T pair is the binding of dGTP (a G-containing nucleotide) in the polymerase's active site. This is followed by the enzyme's catalytic step, in which the DNA is elongated through incorporation of a new $\mathrm{G}$. T base pair.
Once dGTP is bound in the active site, the base pair formed between dGTP and T on the complementary strand assumes a distorted wobble conformation, but seemingly cannot make the conformational transition needed for the catalytic step ${ }^{10}$.

Kimsey and colleagues' current study goes straight to the heart of the mutagenesis model by integrating structural analysis of G.T base pairs in duplexes with measurements of the kinetics of DNA polymerase reactions and computer modelling to show that tautomerism does indeed account for the misincorporation of base pairs. To ensure efficient catalysis, DNA polymerases require optimal geometrical alignment of nucleotide substrates with amino-acid residues in their active site ${ }^{13,14}$. Such alignment can occur when G.T adopts one of its Watson-Crick-like structures (one of the disfavoured tautomeric forms, or the ionized structure ${ }^{15}$ ). Kimsey et al. deduced from their studies that, at neutral $\mathrm{pH}$, at least $99 \%$ of G.T misincorporation is attributable to the formation of G.T tautomers rather than of the ionized structure - from an initially bound G.T wobble pair.

By successfully identifying a role for the disfavoured tautomeric forms of G.T in base-pair misincorporation, Kimsey and colleagues have solved half of the mystery of spontaneous mutagenesis. A solution for the other half now requires the disfavoured tautomeric forms of C.A to be characterized in duplexes and correlated with the rate of C.A misincorporation. So far, NMR and X-ray data have identified only charged $\mathrm{C} \cdot \mathrm{A}^{+}$wobble structures in a DNA duplex ${ }^{7,8}$.

A related challenge would be to establish the mechanism by which 2-aminopurine, a base analogous to both adenine and guanine, induces mutagenesis. For example, 2 -aminopurine is a potent mutagen of the virus bacteriophage $\mathrm{T} 4$, for which it increases the frequency of A.T to G.C mutations (and of the reverse $\mathrm{G} \cdot \mathrm{C}$ to $\mathrm{A} \cdot \mathrm{T}$ mutations) to 10-50 times the frequency of spontaneous mutation levels ${ }^{16}$. If 2 -aminopurine was found to undergo a tautomeric shift much more frequently than A, it would implicate tautomerization in the mechanism, and thus provide the icing on the cake for the tautomerization model of mutagenesis.

Myron F. Goodman is in the Molecular and Computational Biology Program, Departments of Biological Science and Chemistry, University of Southern California, Los Angeles, Los Angeles, California 90089, USA.

e-mail:mgoodman@usc.edu

1. Kimsey, I. J. et al. Nature 554, 195-201 (2018).

2. Watson, J. D. \& Crick, F. H. C. Nature $171,737-738$ (1953).

3. Watson, J. D. \& Crick, F. H. C. Cold Spring Harb. Symp. Quant. Biol. 18, 123-131 (1953).

4. Crick, F. H. C. J. Mol. Biol. 19, 548-555 (1966)

5. Hoogsteen, K. Acta Crystallogr. 16, 907-916 (1963)

6. Sowers, L. C., Eritja, R., Kaplan, B., Goodman, M. F. \& Fazakerly, G. V. J. Biol. Chem. 263, 14794-14801 (1988).

7. Sowers, L. C., Fazakerley, G. V., Kim, H., Dalton, L. \& Goodman, M. F. Biochemistry 25, 3983-3988 (1986).

8. Hunter, W. N., Brown, T., Anand, N. N. \& Kennard, O. Nature 320, 552-555 (1986).

9. Wang, W., Hellinga, H. W. \& Beese, L. S. Proc. Natl Acad. Sci. USA 108, 17644-17648 (2011).

10. Bebenek, K., Pedersen, L. C. \& Kunkel, T. A. Proc Natl Acad. Sci. USA 108, 1862-1867 (2011).

11.Kunkel, T. A. \& Bebenek, K. Annu. Rev. Biochem. 69, 497-529 (2000).

12. Kimsey, I. J., Petzold, K., Sathyamoorthy, B., Stein, Z. W. \& Al-Hashimi, H. M. Nature 519, 315-320 (2015).

13. Echols, H. \& Goodman, M. F. Annu. Rev. Biochem. 60, 477-511 (1991)

14. Tsai, Y.-C. \& Johnson, K. A. Biochemistry 45, 9675-9687 (2006).

15. Yu, H., Eritja, R., Bloom, L. B. \& Goodman, M. F. J. Biol. Chem. 268, 15935-15943 (1993).

16.Drake, J. W. \& Allen, E. F. Cold Spring Harb. Symp. Quant. Biol. 33, 339-344 (1968).

This article was published online on 31 January 2018

\section{CORRECTION}

The News \& Views 'Strategy for making safer opioids bolstered' by Susruta Majumdar and Lakshmi A. Devi (Nature 553, 286-288; 2018) incorrectly stated that more than 100,000 adults suffer from chronic pain in the United States. The correct figure is more than 100 million adults. 